\title{
Inulin results in increased levels of $\beta$-catenin and cyclin D1 as the adenomas increase in size from small to large in the Min/+ mouse
}

\author{
Marjo Misikangas ${ }^{1}$, Heidi Tanayama ${ }^{1}$, Johanna Rajakangas ${ }^{1}$, Jere Lindén ${ }^{2}$, Anne-Maria Pajari ${ }^{1}$ \\ and Marja Mutanen ${ }^{1 *}$ \\ ${ }^{1}$ Department of Applied Chemistry and Microbiology, Division of Nutrition, P.O. Box 66, 00014 University of Helsinki, Finland \\ ${ }^{2}$ Department of Food and Environmental Hygiene, P.O. Box 66, 00014 University of Helsinki, Finland
}

(Received 29 April 2007 - Revised 3 August 2007 - Accepted 16 August 2007 - First published online 31 October 2007)

The mechanism that drives the growth of some colonic adenomas towards malignancy, while permitting others to remain for decades in quiescence, remains unknown. Diets can alter the growth rate of intestinal tumours but it is still unknown whether diets are able to alter the molecular biology of these adenomas in a way that predicts further outcome. To address this issue we fed Min/ + mice with two diets known to lead to different adenoma outcomes: a high-fat control diet $(n 15)$ or a high-fat inulin-enriched $(10 \% \mathrm{w} / \mathrm{w})$ diet $(n 13)$. To study the effect of diet on cell signalling during adenoma growth, the adenomas of each Min/+ mouse were divided into three size-categories, and the levels of $\beta$-catenin, E-cadherin, cyclin D1 and matrix metalloproteinase-9, which are known to be involved in colon tumorigenesis, were determined. The growth-promoting inulin diet resulted in more large adenomas than the control feeding $(P=0.003)$ and doubled the total area of the adenomas $(P=0.008)$. The inulin diet increased the expression of nuclear $\beta$-catenin $(P=0.004)$ and its target cyclin D1 $(P=0.017)$ as the adenomas increased in size from small to large, indicating the presence of an accelerated cancerous process. Neither phenomenon was seen in the control group during adenoma growth. Our results suggest that in addition to the number, size, and growth rate of adenomatous polyps, the signalling pattern of the adenomas should also be considered when evaluating preventive dietary strategies.

Intestinal tumorigenesis: Adenoma growth: E-cadherin: Adenomatous polyposis coli: Colon cancer

The incidence of colon cancer is increasing in the Western world and death from this malignancy is already the second leading cause of cancer mortality in the $\mathrm{USA}^{(1)}$. Ageing of the population and a Western-type life style, including dietary habits and low levels of physical activity, are the main reasons for this incidence. Progression of the disease from benign adenoma through early carcinoma to malignant metastatic cancer requires years or even decades. Due to a long asymptomatic period, all too often the disease is life-threatening before it is diagnosed. Every second person is estimated to have a benign colonic tumour during his or her lifetime and $10 \%$ of these tumours are assumed to progress to malignancy ${ }^{(2)}$. Thus, for preventive strategies it is vitally important to understand the mechanisms that stimulate adenoma growth and development towards accelerated malignancy or, in contrast, attenuate them to remain in quiescence for periods as long as decades.

Several animal models have shown that diet or its constituents can alter the growth rate of intestinal tumours ${ }^{(3)}$. Whether diet is able to alter the molecular biology of these adenomas, which could result in very early prediction of their eventual outcome, is however not known. To address this issue we continued with our previous study ${ }^{(4)}$ where multiple intestinal neoplasia $(\mathrm{Min} /+)$ mice were fed with a high-fat non-fibre diet and a fructo-oligosaccharide inulin diet that led to different adenoma outcomes. We focused on the adenomas and studied whether inulin-induced adenomas were merely larger than the controls or whether the cellular signalling changed during growth of the adenomas. We analysed the level and localisation of cell-signalling proteins in adenomas of three size-categories to determine whether diets regulate the molecular biology of adenomas differently. The signalling proteins chosen were $\beta$-catenin, E-cadherin, cyclin D1, and matrix metalloproteinase-9 (MMP-9). These proteins are either related in adenomatous polyposis coli (APC) protein function ( $\beta$-catenin, E-cadherin, cyclin D1) or predict malignant transformation (MMP-9), and their role in the progression of colon carcinogenesis has been well documented.

During tumorigenesis one of the main functions of the APC protein is disturbed, i.e. regulation of the level and localisation of $\beta$-catenin in the cell ${ }^{(5)}$. In cell membranes $\beta$-catenin forms part of the adherens junctions regulating cell-cell adhesion and connecting the actin cytoskeleton to E-cadherin ${ }^{(6)}$. Normally, the levels of $\beta$-catenin in the cytoplasm or nucleus are kept down by targeting excess $\beta$-catenin to APC-mediated degradation. Activation of the Wnt pathway or a defect in $\beta$-catenin degradation ( $A P C$ mutations) results in stabilisation of cytoplasmic $\beta$-catenin. The stabilised $\beta$-catenin translocates 
into the nucleus where it interacts with the transcriptional factor Tcf/Lef to activate transcription of Wnt-responsive genes, such as the regulators of cell cycle progression: cyclin Dl and $m y c^{(7-9)}$. Furthermore, nuclear staining of $\beta$-catenin correlates strongly with tumour size and dysplasia in human subjects $^{(10-12)}$. Changes in E-cadherin and cyclin D1 expressions were also described in a wide spectrum of human cancers, including colon adenomas and tumours ${ }^{(13-19)}$. Tumour-infiltrating inflammatory cells produce high levels of MMP-9, which are increased early in the transition from colon adenoma to adenocarcinoma ${ }^{(20,21)}$.

\section{Experimental methods}

\section{Study protocol}

Mice were bred at the Laboratory Animal Centre of the University of Helsinki according the study protocol that was approved by The Laboratory Animal Ethics Committee of the University of Helsinki and was published earlier ${ }^{(4)}$. The previous paper presented the cellular signalling in the normal-appearing mucosa of Min/+ mice (C57BL/6J Min/+) at different time points induced by control or inulin diet ${ }^{(4)}$. The present study concentrated on the cellular signalling in the adenomas of 15 -week-old mice analysed by Western blotting. In addition, ten mice were fed either the control diet (five mice) or inulin diet (five mice) from 5 to 14 weeks of age for immunohistochemical and histological studies. These mice were kept in the same conditions as the mice in the main study ${ }^{(4)}$. Both diets provided similar amounts of protein, fat, vitamins, and minerals on an energy basis as described $^{(4)}$. The control diet was a modified high-fat AIN93-G diet ${ }^{(22)}$ with no added fibre. The inulin diet was similar to the control diet but contained $10 \%(\mathrm{w} / \mathrm{w})$ inulin (polydisperse $\beta(2-1)$ fructan, RaftilineHP ${ }^{\circledR}$; Orafti, Tienen, Belgium).

\section{Tissue samples}

The mice were killed at 15 weeks of age by $\mathrm{CO}_{2}$ inhalation. The intestinal tracts were removed, opened longitudinally, and washed with ice-cold $0.9 \% \mathrm{NaCl}$ solution. The small intestine was divided into five equal sections; the caecum and colon were kept together. Two observers blinded to the dietary treatment measured the diameter of all adenomas in each section using a dissecting microscope under $\times 67$ magnification. The adenomas in each section were categorised as small (diameter $<1.1 \mathrm{~mm})$, medium $(1.1-1.5 \mathrm{~mm})$ and large $(>1.5 \mathrm{~mm})$. The adenomas were excised and pooled from each section separately, depending on the size category. During the procedure the samples were kept on ice and only during adenoma enumeration were they exposed to room temperature. Since the intestine was divided into several parts, enumeration of each section was quick and sample degradation minimal. This was ensured by testing samples after several standing times (data not shown). The smallest adenomas that we were able to score had diameters of $0.3 \mathrm{~mm}$. In unclear situations, any adenomas smaller than this were removed but were not included in the adenoma sample. All samples were frozen in liquid $\mathrm{N}_{2}$ and stored at $-70^{\circ} \mathrm{C}$.

\section{Sample preparation}

Sample preparation and Western blotting are described in detail in Misikangas et al. ${ }^{(4)}$. Briefly, all adenomas in the three most distal parts of the small intestine of each animal were pooled according to size category. Each pooled sample consisted all adenomas the mouse had in the size-group (approximately 10-30 adenomas). Samples of small, medium and large adenomas were further fractionated into nuclear, cytosolic, and membranous pools individually for mice, so that each mouse had a total of nine distinct samples. For cellular fractionation various centrifugal forces were used: $15000 \mathrm{~g}$ for the nuclear fraction, $100000 \mathrm{~g}$ for the cytosolic, and Triton X-100 $+100000 \mathrm{~g}$ for the membranous fraction. All fractions were concentrated using Amicon Ultra-4 Centrifugal filter devices (Millipore, Bedford, MA, USA). The purity of the cellular fractions was controlled by determining nuclear lamin B (Sc-6216, Santa Cruz Biotechology, Santa Cruz, CA, USA) levels in the cellular fractions. Both the cytosol and membrane fractions were free of lamin B.

\section{Western blotting}

For $\beta$-catenin all three cellular fractions and for E-cadherin the membranous fractions were used. Cyclin D1 was determined from the cytosol and nucleus. MMP-9 was analysed from the cytosol because this fraction also contains the free extracellular proteins in addition to the actual intracellular cytosolic proteins. For the immunoblot analysis the following primary antibodies were used: anti- $\beta$-catenin (Sc-7199, rabbit polyclonal, Santa Cruz Biotechology), anti-cyclin D1 (RM-9104, rabbit monoclonal, NeoMarkers, Lab Vision Corporation, Fremont, CA, USA), anti-E-cadherin (610182, mouse monoclonal, BD Transduction, San Diego, CA, USA), anti-MMP-9 (M9555, rabbit polyclonal, Sigma-Aldrich Inc, St Louis, MO, USA). Protein detection was carried out using secondary antibodies (Santa Cruz Biotechology): anti-rabbit-HRP (Sc-2030), anti-mouse-HRP (Sc-2031), anti-mouse (Sc-2005), and the enhanced chemiluminescence (ECL and ECL-Plus) detection system (Amersham Corp., Arlington Heigths, IL, USA). The blots were scanned and analysed using a GS-710 Calibrated Imaging Densitometer and the Quantity One program (Bio-Rad Laboratories, Hercules, CA, USA).The results are expressed as sample band intensity (optical density of the protein band multiplied by the band area) divided by the band intensity of the positive control (rat brain, RAW (mouse leukaemic monocyte macrophage cell line), TMK (gastric tumour cell line), A431 (human epithelial carcinoma cell line) or human cells-12 lysates). Equal loading of samples was ensured by incubating blots with $\beta$-actin antibody (A5441, Sigma-Aldrich). Blocking peptides, immunoprecipitation, other commercially available antibodies, or normal serum were used to ensure detection of the correct $\beta$-catenin, cyclin D1, E-cadherin, and MMP-9 bands (data not shown).

\section{Immunohistochemistry and histology}

To confirm the Western blotting results by immunohistochemistry (IHC) and to obtain histological samples of the adenomas, we fed five mice with the control diet and five mice with the inulin diet from 5 to 14 weeks of age. After the feeding period, representative tissue samples of variously 
sized adenomas were taken from the distal small intestine and fixed in phosphate-buffered $4 \%$ paraformaldehyde solution overnight. The fixed tissues were dehydrated, embedded in paraffin, cut to serial $5 \mu \mathrm{m}$ sections and mounted on slides. About three sections per tissue sample, enclosing characteristic adenomatous areas, were selected for IHC and two for histology. For IHC the endogenous peroxidase activity of deparaffinised and rehydrated sections was quenched in $0.9 \% \quad \mathrm{H}_{2} \mathrm{O}_{2}$. The slides were rinsed in Tris-buffered saline, and an antigen retrieval step was carried out in a microwave oven for $15 \mathrm{~min}$ in citrate buffer, $\mathrm{pH}$ 6.0. Immunostaining with anti- $\beta$-catenin (Transduction Laboratories, Lexington, KY, USA) at a 1:3000 dilution, anti-E-cadherin (Transduction Laboratories), at a 1:500 dilution, anti-cyclin D1 (NeoMarkers) at a 1:100 dilution was performed using a Homo-mouse ${ }^{\mathrm{TM}}$ Poly-HRP IHC Detection Kit (KDM-7DAB, ImmunoVision Technologies Company, Brisbane, CA, USA). Negative control tissues were prepared in the same manner, except that the primary antibody was replaced with a negative control for the mouse $\mathrm{IgG}_{2 \mathrm{a}} \mathrm{Ab}-1$ (NeoMarkers). All IHC sections were counterstained with Mayer's hemalaum (Merck, Darmstadt, Germany). For histology, the deparaffinised and rehydrated sections were stained with haematoxylin and eosin.

\section{Immunohistochemical and histological evaluation of} adenomas

Two observers blind to the dietary treatment evaluated the IHC stainings. The $\beta$-catenin, E-cadherin, and cyclin D1 proteins were scored semiquantitatively based on the staining intensity (grade $0=$ negative; grade $1=$ weak; grade $2=$ moderate; grade $3=$ strong) and distribution. The percentage of cells with membranous or cytosolic positivity was graded as follows: 0 $(<5 \%), 1(5-30 \%), 2(30-60 \%), 3(>60 \%)$, and nuclear positivity: $0(<5 \%), 1(5-15 \%), 2(15-50 \%), 3(>50 \%)$. A total score was obtained for each case by multiplying the two respective scores.

Histology of the adenomas was assessed from haematoxylin and eosin stained sections by a veterinary pathologist (J. L.) blinded to the dietary treatment. Special emphasis was placed on dysplasia (growth pattern and differentiation of the neoplastic epithelium) as well as cellular anaplasia (nuclear and cellular morphology and nucleus to cytoplasm ratio) of the adenomas. These were both graded separately according to the following five-tier system: grade $1=$ minimal; grade $2=$ mild; grade $3=$ moderate; grade $4=$ marked; grade $5=$ severe. The amount of adenomas (adenomatous areas) in the studied tissue samples varied from one to seven, thus the most pronounced changes in each sample were used for grading.

\section{Statistical analyses}

The results are expressed as the median and range. The differences in adenoma number and size between the control and inulin groups were analysed with the Mann-Whitney U test. Spearman correlation was used for correlation analysis and the Wilcoxon signed rank test for paired comparisons (StatView, version 5.0.1, SAS Institute Inc., Cary, NC, USA). After a log exchange the diet effect during adenoma growth (small, medium, and large adenomas) on the measured parameters was studied. The diet groups were presented in separate curves that were tested with linear mixed models for repeated measures. Additionally, analysis was adjusted for the number of adenomas (SAS, version 8.2). A difference of $P<0.05$ was considered significant.

\section{Results}

\section{Evaluation of adenomas}

The adenomas in the inulin group were larger than those of the control group (1.3-fold increase, $P=0.0004$; Fig. 1 (C)) but the actual number of adenomas between the groups did not differ (Fig. 1 (A)). Since nearly $80 \%$ of tumours in Min/+ mice develop in the distal part of the small intestine, the result are shown from this area where also the molecular biology was done. Inulin feeding produced more large adenomas (diameter $>1.5 \mathrm{~mm}$ ) than the control feeding (median 15 (range 2-43) inulin $v$. median 5 (range 0-18) control, $P=0.003$; Fig. 1 (C)) but fewer small adenomas (diameter $<1.1 \mathrm{~mm}$ ) than the control (median 11 (range 6-22) inulin $v$. median 21 (range $10-32$ ) control, $P=0.005$ ) in the distal small intestine. The number of medium-sized (diameter $1.1-1.5 \mathrm{~mm}$ ) adenomas was similar in both treatment groups.

To confirm the increased adenoma burden in the inulin group, the area of the adenomas was calculated based on the diameter and number of adenomas. The area of the adenomas was increased by $100 \%$ in the inulin group compared with the control group (median 68.2 (range $21 \cdot 0-140 \cdot 1$ ) $\mathrm{mm}^{2}$ inulin $v$. median 32.5 (range 4.3-97.6) $\mathrm{mm}^{2}$ control, $P=0.008$; Fig. 1 (D)). The greater portion of adenomas in the inulin group was categorised as large and, therefore, their total area was larger than in the same category of the control group (median 43.7 (range $5.1-132.8$ ) $\mathrm{mm}^{2}$ inulin $v$. median 13.2 (range 0-39.6) $\mathrm{mm}^{2}$ control, $P=0.003$; Fig. 1 (D)). On the other hand, $50 \%$ of the adenomas in the control group remained small, in contrast to $25 \%$ in the inulin group.

Histologically the adenomatous areas showed a general sessile or plaquelike pattern, consisting of disorganised intestinal glands forming tortuous tubular structures. The adenomas were accompanied by mild chronic inflammation composed of lymphocytes, some macrophages, and occasional plasma cells and eosinophilic granulocytes. The epithelium of the neoplastic glands was in general moderately (grade 3) dysplastic; mild (grade 2) and marked (grade 5) changes were detected in only two of the twenty slides examined. The anaplasia in all adenomatous areas but one was moderate (grade 3). The histologically discernible nuclear patterns of the tumour cells were therefore remarkably similar in all adenomatous areas studied, regardless of their size or the treatment of the mice.

\section{Amount of $\beta$-catenin and E-cadherin}

To determine the effect of diet on cell signalling during adenoma growth, the adenomas of each Min/ + mouse were divided into three size-categories, and in each the relevant $\beta$-catenin signalling proteins were determined individually for all mice. The main results are presented in Table 1 and for clarity in Fig. 2. The diets had different 
(A)

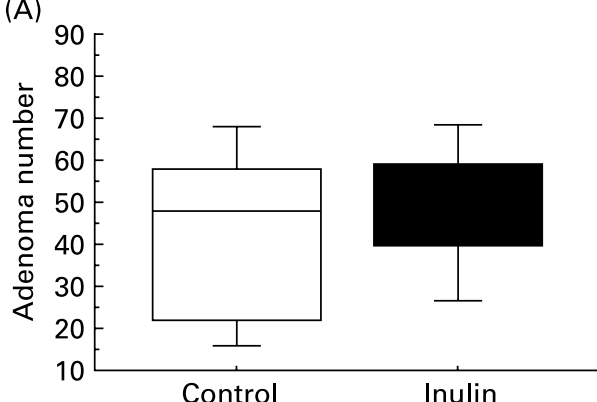

(C)

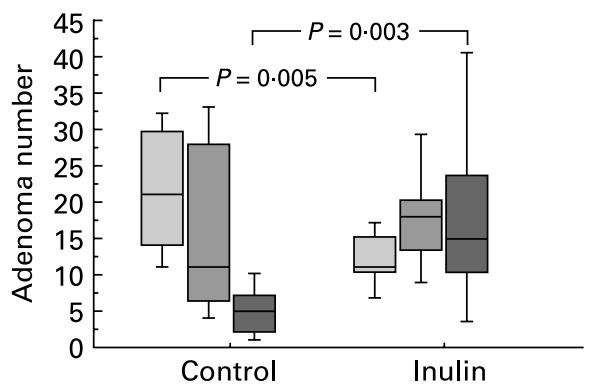

(B)

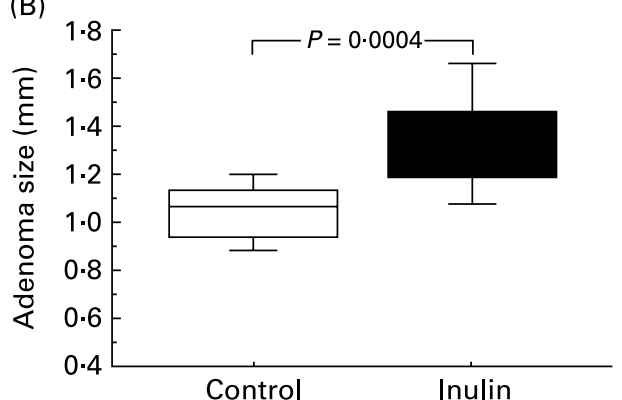

(D)

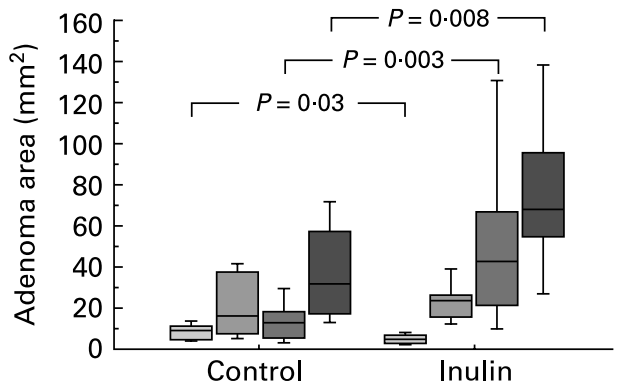

Fig. 1. Total number (A) and size (diameter, mm) (B) of adenomas in the distal small intestine of 15-week-old Min/+ mice fed either a control diet ( $n$ 15) or a diet enriched with $10 \%$ inulin $(n 13)$. Number $(C)$ and area $\left(\mathrm{mm}^{2}\right)(D)$ of adenomas in different size-groups $(\square$, small $(<1.1 \mathrm{~mm})$; $\square$, medium $(1.1-1.5 \mathrm{~mm})$; $\square$, large $(>1.5 \mathrm{~mm})$ ), total) and $\square$, the total area of adenomas in the distal small intestine. Results are presented as box-plots. The box represents the interquartile range, which contains $50 \%$ of the values. The vertical bars extend from the box to the 10th and 90th percentiles of the variable. Medians are indicated by lines across the boxes. $P$ is a comparison by the Mann-Whitney test.

effects on $\beta$-catenin and E-cadherin (Table 1 , diet $\times$ size interactions, $P=0.009-0.036)$. During the inulin diet the amount of $\beta$-catenin in the nucleus increased as the adenomas enlarged (Fig. 2 (A), Wilcoxon signed rank test, paired comparison, $P<0.05$ and Table 1, linear mixed model, inulin diet, small-medium-large adenomas, $P=0.004)$. This phenomenon was not seen in the control diet. Similarly, both the membranous $\beta$-catenin and E-cadherin increased during inulin-induced adenoma growth (Table 1, $P=0.001$ and $P=0.003$, Fig. 2 (B)). In the control group, the amount of membranous E-cadherin remained steady during the adenoma growth and membranous $\beta$-catenin increased only slightly when small and medium adenomas were compared (Table 1, $P=0.026$ ). The Western blotting results were confirmed by IHC staining that showed a tendency for nuclear $\beta$-catenin, as well as membranous $\beta$-catenin and E-cadherin, to increase in the large adenomas of the inulin-fed mice (Fig. 3).

Table 1. Effects of diet on $\beta$-catenin, E-cadherin, cyclin D1, and matrix metalloproteinase (MMP)-9 during adenoma growth were tested, using linear mixed models for repeated measures data $(P$ values). The specific diet effects were found in $\beta$-catenin, E-cadherin, and cyclin D1 (diet $\times$ size interaction $P<0.05)$. The inulin diet increased the amount of nuclear $\beta$-catenin $(P=0.004)$ and also membranous $\beta$-catenin $(P<0.001)$ and $\mathrm{E}$-cadherin $(P=0.003)$ as the adenomas enlarged

\begin{tabular}{|c|c|c|c|c|c|c|c|}
\hline & $\begin{array}{l}\beta-C a t e n i n \\
\text { nucleus }\end{array}$ & $\begin{array}{l}\beta \text {-Catenin } \\
\text { membrane }\end{array}$ & $\begin{array}{l}\text { E-Cadherin } \\
\text { membrane }\end{array}$ & $\begin{array}{l}\text { Cyclin D1 } \\
\text { cytosol }\end{array}$ & $\begin{array}{l}\text { Cyclin D1 } \\
\text { nucleus }\end{array}$ & $\begin{array}{l}\beta-C a t e n i n \\
\text { cytosol }\end{array}$ & $\begin{array}{l}\text { MMP-9 } \\
\text { cytosol }\end{array}$ \\
\hline \multicolumn{8}{|l|}{ Diet-specific interactions } \\
\hline \multicolumn{8}{|l|}{ Difference between the diets } \\
\hline in the small adenomas & 0.031 & 0.047 & 0.072 & NS & & & \\
\hline in the medium adenomas & NS & NS & NS & NS & & & \\
\hline in the large adenomas & NS & 0.013 & NS & NS & & & \\
\hline \multicolumn{8}{|c|}{ Difference between the adenoma size-groups* } \\
\hline control diet: S-M-L & NS & 0.026 & NS & NS & & & \\
\hline S-M & & 0.019 & & & & & \\
\hline S-L & & NS & & & & & \\
\hline$M-L$ & & NS & & & & & \\
\hline inulin diet: S-M-L & 0.004 & $<0.001$ & 0.003 & $<0.001$ & & & \\
\hline S-M & 0.064 & $<0.001$ & 0.004 & $<0.001$ & & & \\
\hline S-L & 0.001 & $<0.001$ & 0.012 & 0.010 & & & \\
\hline$M-L$ & NS & NS & NS & NS & & & \\
\hline
\end{tabular}

*Adenoma size-groups: S, small; M, medium; L, large adenomas. 

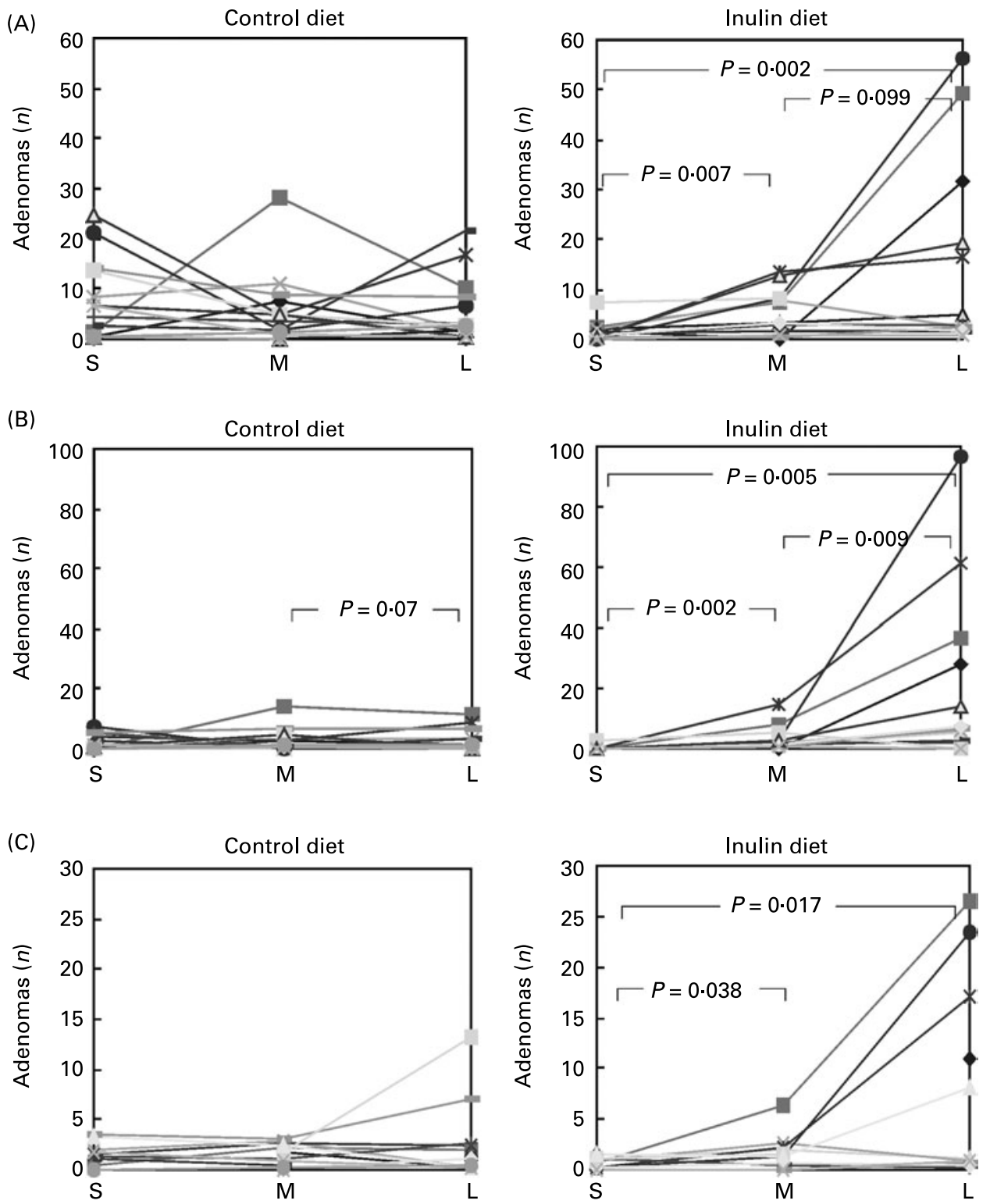

Fig. 2. Amount of $(A)$ nuclear and (B) membranous $\beta$-catenin and $(C)$ nuclear cyclin $D 1$ in adenoma size-groups (S, small; M, medium; L, large) analysed by Western blotting. For the control and inulin groups, the results are presented separately for each mouse and the lines show the change during adenoma growth. Each datapoint is a pooled sample that consists of all adenomas the mouse had in the size-group (approximately 10-30 adenomas). The difference between the adenoma size-groups was tested with the Wilcoxon signed rank test ( $P$ values).

\section{Amount of cyclin D1}

The amount of cyclin D1 increased during inulin-induced adenoma growth, similarly to the increased levels of nuclear $\beta$-catenin, (Fig. $2(\mathrm{C})$, Wilcoxon signed rank test, $P=0 \cdot 017-$ 0.046 and Table 1, linear mixed model, inulin diet, smallmedium-large adenomas, $P<0.001$ ). This was also visible in the IHC-stained large adenomas of the inulin group (Fig. 3). In the control group, the amount of cyclin D1 remained unaltered during adenoma growth (Fig. $2(\mathrm{C})$ ). The amount of nuclear cyclin D1 could have been a consequence of the change in nuclear $\beta$-catenin, since both were strongly correlated $(r$ 0.8, $P<0 \cdot 0001)$. MMP-9 was analysed to determine the presence of any possible shift toward a malignant phenotype, but the MMP-9 levels remained unaltered in both diet groups.

\section{Discussion}

It has been estimated that interventions that decrease the growth rate of adenomatous polyps are much more effective in reducing the risk of colon cancer than those that decrease the rate of mutations at the $A P C$ locus ${ }^{(23)}$. Thus, it is important to understand the early markers for accelerated growth process of adenomas and to be able to evaluate the quality of adenomas with regard to their possible future aggressiveness.

Earlier we have shown that dietary interventions are able to cause changes in cell signalling already in the normal appearing mucosa ${ }^{(4)}$. Here we showed that the tumour growth-promoting inulin diet that doubled the total area of the adenomas also reinforced the early expression of nuclear $\beta$-catenin and cyclin D1 in the adenomas of Min/+ mice. The study was not able to distinguish if the increased cell 


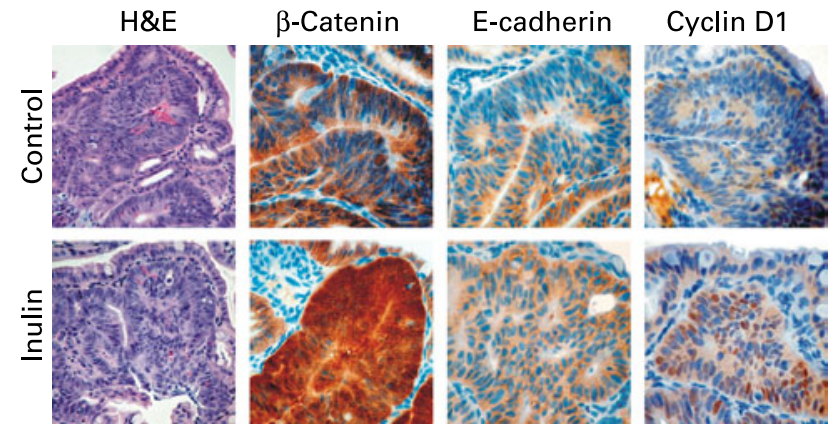

Fig. 3. Representative histological haematoxylin and eosin (H\&E) and immunohistochemical staining of $\beta$-catenin, E-cadherin, and cyclin D1 in the large adenomas of control or inulin-fed mice. Histologically the adenomas were remarkably similar. In the inulin group, the nuclear $\beta$-catenin, membranous E-cadherin, and nuclear cyclin D1 tended to increase. Positive cells show brown staining, $\times 200$ magnification in $\mathrm{H} \& \mathrm{E}, \times 400$ magnification in immunohistochemical staining.

signalling was a cause or a consequence of rapid adenoma growth. The growth of the adenomas and the concomitant increase in $\beta$-catenin and cyclin D1 by inulin is a very important result in the light of possible preventive dietary strategies. Our study clearly shows that dietary interventions at the same genetic background ( $\mathrm{Apc}^{-/-}$tissue) are able to alter the cellular signalling of the adenomas, i.e. to result in qualitative differences that may predict the future outcome of the adenomas.

In chemically induced colon cancer models inulin has not shown similar growth-promoting effect as found in our study, actually the opposite has been found ${ }^{(24)}$. When comparing the results between $\mathrm{Min} /+$ mice and chemical rodent model one should keep in mind that different models present different stages of colorectal tumorigenesis which can influence the chemoprevention. In addition, molecular genetics of chemical rodent models are different from that found in human colon tumorigenesis. Azoxymethane causes $\beta$-catenin and K-ras mutations ${ }^{(25)}$ that are usually found in the later stages of human adenoma-carcinoma sequence. For example mutation in $A p c$, the earliest change found in inherited familial adematous polyposis as well as in most of sporadic colon cancers, is less frequent and mutation in p53 is totally absent in chemically induced rodent models ${ }^{(25-27)}$. The type and chain length of inulin may also be important. Bearing this in mind it must be emphasised that this study was conducted in Min/+ mice and its validity in human subjects has to be studied further. However, results obtained from polyp and colon cancer patients receiving inulin as a part of a symbiotic diet suggested that the effect of inulin may be dependent on the stage of preneoplastic lesion and genetic background $^{(28)}$ which emphasises the importance of using also Min/+ mice when studying inulin.

$\mathrm{Wnt} / \beta$-catenin signalling is crucial to the initial expansion of the transformed cell compartment and is also involved in tumour progression. Staining for $\beta$-catenin in colorectal carcinomas often shows a heterogeneous pattern with strong nuclear enrichment at the invasive front and mainly cytoplasmic and membranous staining in the central tumour area $^{(29)}$. Nuclear $\beta$-catenin, which may be the key player in transition to the invasive state of the tumour, has several target genes that regulate tumour proliferation, malignancy, and invasiveness. In human subjects the amount of nuclear $\beta$-catenin increases from early adenomas to adenocarcinomas ${ }^{(10-12)}$. Here we showed in Min/+ mice that during inulin diet the amount of nuclear $\beta$-catenin increased as adenomas enlarged, which may indicate diet-specific acceleration in tumour progression.

Cyclin D1, one target of $\beta$-catenin and a positive effector of the cell cycle, is frequently overexpressed in a broad range of human tumour types, including colon cancer $^{(14-19)}$. Nuclear staining of cyclin D1 increases significantly from low-grade dysplastic adenomas to high-grade dysplasia ${ }^{(30)}$ and it also correlates with the early onset of cancer and risk of tumour progression and metastasis ${ }^{(14-19)}$. However, new studies claimed that cyclin D1 is not the immediate target of $\beta$-catenin and that the up regulation of cyclin D1 in intestinal neoplasia may be an important factor in adenoma establishment and growth rather than initiation ${ }^{(31)}$. Some authors also proposed that cyclin D1 may not be essential for the development of intestinal tumorigenesis but may modify adenoma frequency $^{(32,33)}$. Our results showed that during inulin diet the amount of cyclin D1 increased as adenomas enlarged and also revealed the importance of nuclear cyclin D1 during tumour progression.

The loss of E-cadherin may release $\beta$-catenin from cell membranes and increase the cytoplasmic pool of $\beta$-catenin that is available for nuclear signalling ${ }^{(34,35)}$. Although the reciprocity between membranous and nuclear expression of $\beta$-catenin is known ${ }^{(10)}$, contrasting or neutral findings were also described ${ }^{(11)}$. Participation of $\beta$-catenin in adhesion or Wnt signalling may be dictated by the regulation of distinct molecular forms of $\beta$-catenin with different binding properties, rather than simple competition between cadherins and $\mathrm{T}$-cell factors for a single constitutive form ${ }^{(36)}$. Our findings that the amounts of membranous E-cadherin and $\beta$-catenin increased as the adenomas enlarged could be a result of the overall increase in protein in contrast to increase in some parts of the cell. Our results are in line with reports ${ }^{(37,38)}$ showing that the expression and association of E-cadherin and $\beta$-catenin are increased in $A p c-1-$ adenomas compared with normal-appearing $A p c+/$ - mucosa and may result in tighter adhesion of tumour cells. In tissue culture cells, increased levels of $\beta$-catenin induced by Wnt signalling led to saturation of $\beta$-catenin binding to cadherin at the plasma membrane and increase in cell-cell adhesion ${ }^{(39)}$.

The histology of the adenomas revealed them to be conspicuously similar in their growth pattern as well as the dysplasia and anaplasia stages. All histologically examined tumours were also clearly benign. This is in agreement with the amounts of MMP-9 in the tumours, which did not differ between the diets or adenoma size-groups. The results support the idea that the adenomas of Min/+ mice are still benign and that the MMP-9 gelatinase that increases early in the transition from colon adenoma to adenocarcinoma ${ }^{(20,21)}$ is not yet needed for tumour cell invasion.

Dietary inulin-accelerated adenoma growth and all molecular biological changes occurred earlier in this group, despite the similar histology of the adenomas. Even in the same size-category of adenomas the diets reinforced $\beta$-catenin signalling differently and adenomas expected to behave more aggressively are most likely found in the highest subgroup. 
Our results suggest, that in addition to the number, size, and growth rate of adenomatous polyps, the signalling pattern of the adenomas should also be considered when evaluating preventive dietary strategies.

\section{Acknowledgements}

We thank Maija Järvinen for excellent technical assistance and Pertti Mutanen (Dipper Software) for help with statistical analysis. This study was supported by the Innovation in Food Program of the National Technology Agency of Finland, the Finnish Cultural Foundation (Alma and Jussi Jalkanen Fund), and The Finnish Graduate School on Applied Bioscience.

\section{References}

1. Jemal A, Siegel R, Ward E, Murray T, Xu J \& Thun MJ (2007) Cancer statistics, 2007. CA Cancer J Clin 57, 43-66.

2. Kinzler KW \& Vogelstein B (1996) Lessons from hereditary colorectal cancer. Cell 87, 159-170.

3. Corpet DE \& Pierre F (2003) Point: From animal models to prevention of colon cancer. Systematic review of chemoprevention in min mice and choice of the model system. Cancer Epidemiol Biomarkers Prev 12, 391-400.

4. Misikangas M, Pajari AM, Paivarinta E \& Mutanen M (2005) Promotion of adenoma growth by dietary inulin is associated with increase in cyclin D1 and decrease in adhesion proteins in Min/+ mice mucosa. J Nutr Biochem 16, 402-409.

5. Näthke IS (2004) The adenomatous polyposis coli protein: the Achilles heel of the gut epithelium. Annu Rev Cell Dev Biol 20, 337-366

6. Cowin P (1994) Unraveling the cytoplasmic interactions of the cadherin superfamily. Proc Natl Acad Sci U S A 91, 10759-10761.

7. Shtutman M, Zhurinsky J, Simcha I, Albanese C, D'Amico M, Pestell R \& Ben-Ze'ev A (1999) The cyclin D1 gene is a target of the beta-catenin/LEF-1 pathway. Proc Natl Acad Sci U S A 96, 5522-5527.

8. Tetsu O \& McCormick F (1999) Beta-catenin regulates expression of cyclin D1 in colon carcinoma cells. Nature 398, 422-426.

9. He TC, Sparks AB, Rago C, Hermeking H, Zawel L, da Costa LT, Morin PJ, Vogelstein B \& Kinzler KW (1998) Identification of c-MYC as a target of the APC pathway. Science 281, 1509-1512

10. Hao X, Tomlinson I, Ilyas M, Palazzo JP \& Talbot IC (1997) Reciprocity between membranous and nuclear expression of beta-catenin in colorectal tumours. Virchows Arch 431, 167-172.

11. Herter P, Kuhnen C, Muller KM, Wittinghofer A \& Muller O (1999) Intracellular distribution of beta-catenin in colorectal adenomas, carcinomas and Peutz-Jeghers polyps. J Cancer Res Clin Oncol 125, 297-304.

12. Brabletz T, Herrmann K, Jung A, Faller G \& Kirchner T (2000) Expression of nuclear beta-catenin and c-myc is correlated with tumor size but not with proliferative activity of colorectal adenomas. Am J Pathol 156, 865-870.

13. Cavallaro U \& Christofori G (2004) Cell adhesion and signalling by cadherins and Ig-CAMs in cancer. Nat Rev Cancer 4, $118-132$

14. Arber N, Hibshoosh H, Moss SF, Sutter T, Zhang Y, Begg M, Wang S, Weinstein IB \& Holt PR (1996) Increased expression of cyclin D1 is an early event in multistage colorectal carcinogenesis. Gastroenterology 110, 669-674.

15. Kristt D, Turner I, Koren R, Ramadan E \& Gal R (2000) Overexpression of cyclin D1 mRNA in colorectal carcinomas and relationship to clinicopathological features: an in situ hybridization analysis. Pathol Oncol Res 6, 65-70.

16. Bartkova J, Thullberg M, Slezak P, Jaramillo E, Rubio C, Thomassen LH \& Bartek J (2001) Aberrant expression of G1phase cell cycle regulators in flat and exophytic adenomas of the human colon. Gastroenterology 120, 1680-1688.

17. Utsunomiya T, Doki Y, Takemoto H, Shiozaki H, Yano M, Sekimoto M, Tamura S, Yasuda T, Fujiwara Y \& Monden M (2001) Correlation of beta-catenin and cyclin D1 expression in colon cancers. Oncology 61, 226-233.

18. Bahnassy AA, Zekri AR, El-Houssini S, El-Shehaby AM, Mahmoud MR, Abdallah S \& El-Serafi M (2004) Cyclin A and cyclin D1 as significant prognostic markers in colorectal cancer patients. BMC Gastroenterology 4, 22.

19. Mermelshtein A, Gerson A, Walfisch S, Delgado B, ShechterMaor G, Delgado J, Fich A \& Gheber L (2005) Expression of D-type cyclins in colon cancer and in cell lines from colon carcinomas. Br J Cancer 93, 338-345.

20. Zucker S \& Vacirca J (2004) Role of matrix metalloproteinases (MMPs) in colorectal cancer. Cancer Metastasis Rev 23, $101-117$

21. Mook OR, Frederiks WM \& Van Noorden CJ (2004) The role of gelatinases in colorectal cancer progression and metastasis. Biochim Biophys Acta 1705, 69-89.

22. Reeves PG, Nielsen FH \& Fahey GC Jr (1993) AIN-93 purified diets for laboratory rodents: final report of the American Institute of Nutrition ad hoc writing committee on the reformulation of the AIN-76A rodent diet. J Nutr 123, 1939-1951.

23. Luebeck EG \& Moolgavkar SH (2002) Multistage carcinogenesis and the incidence of colorectal cancer. Proc Natl Acad Sci U S A 99, 15095-15100.

24. Pool-Zobel BL (2005) Inulin-type fructans and reduction in colon cancer risk: review of experimental and human data. Br J Nutr 93, Suppl. 1, S73-S90.

25. Takahashi M \& Wakabayashi K (2004) Gene mutations and altered gene expression in azoxymethane-induced colon carcinogenesis in rodents. Cancer Sci 95, 475-480.

26. Song J, Medline A, Mason JB, Gallinger S \& Kim Y-I (2000) Effects of dietary folate on intestinal tumorigenesis in the ApcMin Mouse. Cancer Res 60, 5434-5440.

27. O'Shaughnessy JA, Kelloff GJ, Gordon GB, et al. (2002) Treatment and prevention of intraepithelial neoplasia: an important target for accelerated new agent development. Clin Cancer Res 8, 314-346.

28. Rafter J, Bennett M, Caderni G, et al. (2007) Dietary synbiotics reduce cancer risk factors in polypectomized and colon cancer patients. Am J Clin Nutr 85, 488-496.

29. Behrens $\mathbf{J}$ (2005) The role of the Wnt signalling pathway in colorectal tumorigenesis. Biochem Soc Trans 33, 672-675.

30. Zhang T, Nanney LB, Luongo C, Lamps L, Heppner KJ, DuBois RN \& Beauchamp RD (1997) Concurrent overexpression of cyclin D1 and cyclin-dependent kinase 4 (Cdk4) in intestinal adenomas from multiple intestinal neoplasia (Min) mice and human familial adenomatous polyposis patients. Cancer Res 57, 169-175.

31. Sansom OJ, Reed KR, van de Wetering M, Muncan V, Winton DJ, Clevers H \& Clarke AR (2005) Cyclin D1 is not an immediate target of beta-catenin following Apc loss in the intestine. J Biol Chem 280, 28463-28467.

32. Wilding J, Straub J, Bee J, Churchman M, Bodmer W, Dickson C, Tomlinson I \& Ilyas M (2002) Cyclin D1 is not an essential target of beta-catenin signaling during intestinal tumorigenesis, but it may act as a modifier of disease severity in multiple intestinal neoplasia (Min) mice. Cancer Res 62, 4562-4565.

33. Hulit J, Wang C, Li Z, et al. (2004) Cyclin D1 genetic heterozygosity regulates colonic epithelial cell differentiation and tumor number in ApcMin mice. Mol Cell Biol 24, 7598-7611. 
34. Smits R, Ruiz P, Diaz-Cano S, Luz A, Jagmohan-Changur S, Breukel C, Birchmeier C, Birchmeier W \& Fodde R (2000) E-cadherin and adenomatous polyposis coli mutations are synergistic in intestinal tumor initiation in mice. Gastroenterology 119, $1045-1053$.

35. Gottardi CJ, Wong E \& Gumbiner BM (2001) E-cadherin suppresses cellular transformation by inhibiting beta-catenin signaling in an adhesion-independent manner. $J$ Cell Biol 153, 1049-1060

36. Gottardi CJ \& Gumbiner BM (2004) Distinct molecular forms of beta-catenin are targeted to adhesive or transcriptional complexes. J Cell Biol 167, 339-349.
37. Carothers AM, Melstrom KA Jr, Mueller JD, Weyant MJ \& Bertagnolli MM (2001) Progressive changes in adherens junction structure during intestinal adenoma formation in Apc mutant mice. J Biol Chem 276, 39094-39102.

38. Ju J, Hong J, Zhou JN, et al. (2005) Inhibition of intestinal tumorigenesis in Apcmin/+ mice by (-)-epigallocatechin-3gallate, the major catechin in green tea. Cancer Res $\mathbf{6 5}$, $10623-10631$.

39. Hinck L, Nelson WJ \& Papkoff J (1994) Wnt-1 modulates cellcell adhesion in mammalian cells by stabilizing beta-catenin binding to the cell adhesion protein cadherin. J Cell Biol 124, 729-741. 